\title{
вмJ Global Health Automated phone call and text reminders for childhood immunisations (PRIMM): a randomised controlled trial in Nigeria
}

\author{
Osayame A Ekhaguere, ${ }^{01}$ Rosena O Oluwafemi, ${ }^{2}$ Bolaji Badejoko, ${ }^{3}$ \\ Lawal O Oyeneyin, ${ }^{4}$ Azeez Butali, ${ }^{5}$ Elizabeth D Lowenthal, ${ }^{6}$ Andrew P Steenhoff ${ }^{7}$
}

To cite: Ekhaguere $\mathrm{OA}$, Oluwafemi RO, Badejoko B, et al. Automated phone call and text reminders for childhood immunisations (PRIMM): a randomised controlled trial in Nigeria. BMJ Glob Health 2019;4:e001232. doi:10.1136/ bmjgh-2018-001232

Handling editor Seye Abimbola

- Additional material is published online only. To view please visit the journal online (http://dx.doi.org/10.1136/ bmjgh-2018-001232).

EDL and APS are joint senior authors.

Received 17 October 2018 Revised 21 January 2019 Accepted 25 January 2019

Check for updates

(c) Author(s) (or their employer(s)) 2019. Re-use permitted under CC BY-NC. No commercial re-use. See rights and permissions. Published by BMJ.

For numbered affiliations see end of article.

Correspondence to Dr Osayame A Ekhaguere; osaekhag@iu.edu

\section{ABSTRACT}

Background Sub-Saharan Africa has high under-5 mortality and low childhood immunisation rates. Vaccinepreventable diseases cause one-third of under- 5 deaths. Text messaging reminders improve immunisation completion in urban but not rural settings in sub-Saharan Africa. Low adult literacy may account for this difference. The feasibility and impact of combined automated voice and text reminders on immunisation completion in rural sub-Saharan Africa is unknown.

Methods We randomised parturient women at the Mother and Child Hospitals Ondo State, Nigeria, owning a mobile phone and planning for child immunisation at these study sites to receive automated call and text immunisation reminders or standard care. We assessed the completion of the third pentavalent vaccine (Penta-3) at 18 weeks of age, immunisation completion at 12 months and within 1 week of recommended dates. We assessed selected demographic characteristics associated with completing immunisations at 12 months using a generalised binomial linear model with 'log' link function. Feasibility was assessed as proportion of reminders received.

Results Each group had 300 mother-baby dyads with similar demographic characteristics. At 18 weeks, 257 (86\%) and 244 (81\%) (risk ratio (RR) 1.05, 95\% $\mathrm{Cl} 0.98$ to $1.13 ; \mathrm{p}=0.15$ ) in the intervention and control groups received Penta-3 vaccine. At 12 months, $220(74 \%)$ and $196(66 \%)$ (RR 1.12, 95\% Cl 1.01 to $1.25 ; p=0.04)$ in the intervention and control groups received the measles vaccine. Infants in the intervention group were more likely to receive Penta-3 (84\% vs $78 \%$, RR $1.09,95 \%$ $\mathrm{Cl} 1.01$ to $1.17 ; \mathrm{p}=0.04$ ), measles ( $73 \%$ vs $65 \%$, RR $1.13,95 \% \mathrm{Cl} 1.02$ to $1.26 ; \mathrm{p}=0.02)$ and all scheduled immunisations collectively ( $57 \%$ vs $47 \%$, RR $1.13,95 \% \mathrm{Cl}$ 1.02 to $1.26 ; p=0.01$ ) within 1 week of the recommended date. No demographic character predicted immunisation completion. In the intervention group, 92\% and $86 \%$ reported receiving a verification reminder and at least one reminder during the study period, respectively.

Conclusion Paired automated call and text reminders significantly improved immunisation completion and timeliness.

Trial registration number NCT02819895.

\section{Key questions}

What is already known?

- In sub-Saharan Africa, text message reminders alone improve childhood immunisation uptake in urban areas but not in rural areas where literacy levels are lower.

- Low adult literacy rates in sub-Saharan Africa may limit the use of stand-alone text messaging reminder systems.

What are the new findings?

- In a semi-rural setting in Nigeria, paired automated voice call and text immunisation reminders significantly improved the completion and timely receipt of immunisations.

- Cost of transportation, distance to and length of time spent at the immunisation clinics were additional significant barriers to immunisation reported in this setting.

\section{What do the new findings imply?}

- Our results add to the body of literature on the impact of phone reminders on immunisation completion and inform research on innovative solutions capable of improving access to and efficiency of immunisation clinics.

\section{INTRODUCTION}

Vaccine-preventable diseases are a leading cause of under-5 mortality. ${ }^{1}$ Sub-Saharan Africa (SSA) and Southeast Asia-regions with poor immunisation uptake and completion rates-carry $80 \%$ of the global under- 5 mortality burden. ${ }^{12}$ These regions contribute significantly to the stalled global immunisation coverage. ${ }^{3}$ Nigeria has the highest number of underimmunised and unimmunised children worldwide. ${ }^{34}$ A 2017 Nigerian National Immunization Coverage Survey estimated that $37 \%$ and $40 \%$ of children in Nigeria aged 12-24 months were underimmunised and unimmunised, respectively. ${ }^{5} \mathrm{~A}$ 
fully immunised child is defined as one who received one Bacille Calmette-Guerin (BCG), three pentavalent (ie, diphtheria, tetanus, pertussis, Haemophilus influenzae type B and hepatitis B), one yellow fever and measles vaccine within the first year of life. The third dose of the pentavalent vaccine (Penta-3) is the global benchmark for vaccine reporting. To meet the 2020 Global Vaccine Action Plan targets, interventions that are innovative, cost-effective, region and country specific are needed.

In high-income countries, immunisation reminders through automated or real-time text and or calls improve immunisation uptake and completion. ${ }^{6}$ According to the Nigerian National Immunization Coverage Survey report, lack of awareness of recommendations for a child's immunisation is the biggest reason for not completing the immunisation series-accounting for $42 \%$ of the reported barriers. ${ }^{5}$ With high mobile phone penetration in Nigeria and other SSA countries, phone reminders have the potential to help overcome this barrier.

Evidence from randomised controlled trials (RCTs) conducted in urban areas of Zimbabwe, Nigeria and Burkina Faso suggest text reminders alone improve immunisation completion and timeliness. ${ }^{7-9}$ However, a recent RCT from rural Kenya contradicts these findings. ${ }^{10}$ In this study, text message alone did not improve immunisation completion, but improved timeliness of vaccine receipt. Only when text reminders were paired with a higher monetary incentive did completion rates improve. ${ }^{10}$ Text reminders assume the recipient is literate enough to read and understand the message. However, $38 \%$ of African adults (some 153 million) are illiterate-two-thirds of these being women. ${ }^{11}$ Adult literacy is often lower in rural compared with urban areas. ${ }^{12} 13$ This may account for the difference in effectiveness-an indication that text reminders alone are not appropriate in this setting. In a study from a rural community in Nigeria, the use of manual phone call immunisation reminders significantly improved immunisation completion-a $41 \%$ increase from controls. ${ }^{14}$ However, this method is time and human resource dependent and unlikely to garner national application. With literacy being a limitation in some areas, automated audible reminders in the native languages may provide added benefit to text reminders.

We conducted the Phone Reminder for Immunisation (PRIMM) trial to test the feasibility and effect of pairing automated text and call reminders on immunisation completion in a semi-rural setting in Nigeria. We hypothesised that the Penta-3 vaccine and the completion of the childhood immunisation series assessed at 12 months of age would significantly improve with this intervention.

\section{METHODS}

\section{Study design}

A two-arm parallel RCT was conducted at the Mother and Child Hospital Ondo Town (MCH-Ondo) and Akure (MCH-Akure), in Ondo State, Nigeria. The conduct, analysis and reporting of results are in accordance with the Consolidated Standards of Reporting Trials guidelines for reporting parallel group randomised trials. ${ }^{15}$

The Nigerian government provides routine childhood immunisation at no cost to recipients. Government-run immunisation clinics are locations where vaccines are routinely received. Dictated by the national programme on immunisation, ${ }^{16}$ the routine immunisation schedule in Ondo State, Nigeria is as follows:

- Birth: BCG, hepatitis B virus and oral polio vaccine (OPV-0).

- Six weeks: Penta-1, OPV-1, pneumococcal conjugate vaccine (PCV-1) and rotavirus vaccine (Rota-1).

- Ten weeks: Penta-2, OPV-2, Rota-2 and PCV-2.

- Fourteen weeks: Penta-3, OPV-3, PCV-3 and inactivated polio vaccine (IPV).

- Six months: vitamin A.

- Nine months: measles and yellow fever vaccine.

Immunisation clinic record-book audits were planned to assess vaccination uptake. However, the study was impacted by a hospital workers' strike lasting 6 weeks (2 February to 15 March 2017). The strike interrupted enrolment, immunisation receipt and monitoring. During this period, parents sought other government and private clinics for immunisations. We therefore amended our study protocol to include phone audits for reporting of immunisation uptake between weeks 18 and 30 of each participant's study enrolment period. This was to limit misclassification of the immunisation endpoint. We incorporated the phone call immunisation audit into the planned mid-study survey. The applicable ethical review bodies approved the amendment.

\section{Study participants and setting}

Parturient women and their healthy newborn infants delivered at MCH-Ondo and Akure were eligible for enrolment. We included mothers of healthy newborn babies, who owned a mobile phone and planned to attend the MCH immunisation clinics. We excluded mothers of ill newborns, multiple births and those without mobile phones.

The MCHs are state-run facilities. They provide free healthcare services to pregnant women and children under age 5 , with most patients being middle-income and low-income families. Each hospital runs its own immunisation clinic. Ondo State is in the southwest region of Nigeria. The main local language is Yoruba. The projected 2016 population size from the 2006 national census for Ondo Town and Akure are 389900 and 486 300 , respectively. ${ }^{17}$ The primary occupations of citizens include farming, artisanship, trading and public service. ${ }^{17}$

\section{Study intervention}

We developed a customised Windows software application (app) designed to send automated voice call text and email immunisation reminders. We integrated a secure cloud communications platform, called Twilio, into the app. Messaging and voice were sent by Twilio through the app. Date of birth of the newborn and the 
phone number of the mother and father, when provided, were inputed into the app. The immunisation reminders were autocalculated from date of birth of the child and tailored to the local immunisation schedule. At enrolment, the registered phone number(s) received a verification message. Thereafter, reminders were sent 2 days and the day before the scheduled date of the Penta- 1 , 2, 3 and measles immunisations at 08:00. Eight sets of reminders were sent to each participant. The delivery of text and call occurred at the same time. Voicemail service was not available during the study period.

The automated text message reminder was in English. The text reminder read, 'Reminder from MCH-Your baby's next immunisation visit is in 2 days (or 1 day as appropriate). Immunisation protects your child against killer diseases. Please bring your baby for this visit'. The automated call reminder was in English and Yoruba. The duration of the call was 50 $\mathrm{s}$, had a $5 \mathrm{~s}$ delay before starting and expressed the same message as the text. It cost US $\$ 0.0075$ to send a text and US $\$ 0.015 / \mathrm{min}$ for an automated call. There was no cost to the recipient. Whether study participants received or read the text message and whether participants listened to audio messages in its entirety could not accurately be determined from our telecommunications platform.

\section{Study procedures}

A research assistant at each site assessed mothers daily in the postpartum ward for eligibility. We systematically recorded the number of screened women and the reason for exclusion; however, due to a clerical error at MCH-Ondo, the exact numbers and reasons are unavailable.

In developing the study protocol, we took into consideration that the shortest interval between the pentavalent vaccines is 3 weeks. In Nigeria, the immunisation schedule allows for only 4 weeks between Penta- 1 and 2, and between Penta-2 and $3 .{ }^{18}$ We anticipated a potential stacking of reminders, and immunisation ineligibility, if there was any delay in receiving a scheduled immunisation greater than 1 week. Hence, a priori, we determined the reminders for Penta- 2 and 3 would be recalculated from the date the Penta- 1 and 2 were administered, respectively. For those who did not receive either Penta-1 and or 2, the Penta-2 and 3 reminders were sent 2 weeks after the Penta- 1 and 2 were past due, respectively. We performed daily audits of the immunisation clinic record book. When vaccines were received later than expected, the vaccine receipt date was used to calculate the next scheduled vaccine accordingly.

A child-health immunisation card, which listed the ages when a child was to receive his/her immunisation, comprised standard care. The intervention group received the automated text and call reminder plus standard care, while the control group received only standard care.

We obtained data for the primary outcome from immunisation clinic record books maintained at immunisation clinics and during the mid-study phone survey of all participants. Study research assistants called each study participant on the telephone and obtained verbal reports-name and date- of when Penta-1, 2 and 3 were received. We assessed the receipt of measles vaccine solely from the immunisation clinic record book. This was because the health workers' strike did not affect the receipt or monitoring of the measles immunisation. We did not physically audit the child-health immunisation card given to parents and caregivers.

\section{Study outcome}

The primary outcome was the proportion of infants who received the Penta-1, 2 and 3 immunisations (henceforth referred to as Penta-3) at 18 weeks of age. The administration of BCG vaccination occurs at hospital discharge. Our intervention did not influence BCG receipt, and so it was not included in our primary outcome. We defined the secondary outcomes as completing Penta- 3 and the measles immunisation by 12 months of age and receiving each within 1 week of the recommended time.

We assessed feasibility by the proportion of participants who received the verification text and call at enrolment, as well as those who reported receiving the reminders during the mid-study survey. Additionally, using a socioecological framework, we designed and administered a pre-study survey to assess sociodemographic characteristics of the mothers. We categorised the survey questions into maternal demographics, knowledge about and attitude towards immunisations, mothers' health and healthseeking behaviour, household demographic construct and access to health facility and health information related to immunisation. The mid-study survey was to assess acceptability of the intervention, perception of phone reminders by both groups and perceived barriers to completing immunisations.

\section{Randomisation and blinding}

Mother-infant dyad assignments to study groups in a 1:1 ratio was by a permuted randomisation scheme, ${ }^{19}$ using balanced random blocks of 6,8 or 10 . We stratified the randomisation by study site to account for centre population differences.

Randomisation was done in May 2016 at The Children's Hospital of Philadelphia. Allocation assignments were stored in sealed opaque envelopes and mailed via courier to the local study principal investigators. Only after obtaining written informed consent did the local study teams know the allocation assignment. Neither study participants nor research team were blinded. However, the immunisation clinic staff-those who administered and recorded immunisation-were blinded to study group allocations.

\section{Sample size and statistical methods}

Based on audits of the 2015 MCH-Ondo immunisation records and statewide reports, the baseline Penta-3 completion rate estimate was $75 \%$. To account for a $10 \%$ loss to follow-up, we needed 300 mother-infant dyads in 
each study arm to have a statistical power of $80 \%$ and an alpha level of 0.05 , to detect a $10 \%$ difference in the primary outcome. We deemed this $10 \%$ difference to be of public health importance.

With the protocol amendment, we defined two study populations for the primary outcome analysis. First, a modified intention-to-treat (mITT) population-defined as all randomised subjects regardless of where immunisation was received or audited-immunisation record books or phone calls. The second was the per-protocol (PP) population-defined as all subjects who received immunisations only at MCH-Ondo or Akure and had immunisation receipt audited solely from the immunisation clinic record books.

We compared demographic characteristics and poststudy survey variables between study groups using standard descriptive statistics. We used two-sample t-test or Wilcoxon rank-sum test for continuous variables and $\chi^{2}$ test or Fisher's exact test for categorical variables. Risk ratios and risk difference were calculated for the primary and secondary outcomes. In a post hoc analysis, we used a generalised linear model for binomial distributions with ' $\log$ ' link function to examine the association of selected demographic characteristics by study group on immunisation completion at 12 months. The selected variables were based on demographic factors reported in the literature to influence immunisation completion. ${ }^{5}{ }^{10-22} \mathrm{We}$ tested interaction effects of the demographic factors with the study group and report the $\mathrm{p}$ values for the interaction effects. Results are expressed as risk ratio along with their corresponding 95\% CIs. Data were analysed using Stata V.15.1 (StataCorp, College Station, Texas, USA) with a two-sided significance level of 0.05 .

\section{RESULTS}

We enrolled 250 mother-infant dyads from MCH-Ondo and 350 from Akure between August 2016 and June 2018. Five $(0.8 \%)$ infants died during the follow-up period, 48 $(8 \%)$ were lost to follow-up for the primary outcome and $184(31 \%)$ for the 12-month outcomes (figure), while $155(26 \%)$ received the Penta-3 immunisation at a different clinic.

\section{Characteristics of study subjects}

Infant and maternal demographic information were similar in both groups (table 1). Newborns were $48 \%$ female; the mean (SD) birth weight was $3040 \mathrm{~g}$ (485) and median (IQR) gestational age at birth was 38 (37-38) weeks, respectively (table 1 ). The majority of mothers were aged 18-35 years $(88 \%)$, married $(98 \%)$, and had a university (58\%) or secondary education $(39 \%)$. There were no differences in maternal knowledge about and attitude towards immunisation, mothers' health and health-seeking behaviour, household demographics or access to health facility and health information related to immunisation (table 1).

\section{Primary and secondary analyses}

For the Penta-3 completion, there was no significant difference between the intervention and control groups in either the mITT population assessed at 18 weeks $(86 \%$ vs $81 \%$, risk ratio (RR) $1.05,95 \%$ CI 0.98 to 1.13 ; $p=0.15$ ) (table 2), 12 months (online supplementary table 1 and figure) or in the PP population (91\% vs $88 \%$, RR 1.03 , $95 \%$ CI 0.97 to $1.10 ; \mathrm{p}=0.37$ ) (online supplementary table 2). The proportion of infants who completed the 12-month immunisation series was significantly higher in the intervention compared with the control group (74\% vs $66 \%$, RR 1.12, 95\% CI 1.01 to $1.25 ; \mathrm{p}=0.03$ ) (table 2). To achieve completion of the immunisation series for one infant, 12.5 parents would need to receive the call and text message reminders. The proportion of infants who received the Penta-3 and measles immunisation within 1 week of the expected date was also significantly higher in the intervention compared with the control groups $(84 \%$ vs $78 \%$, RR $1.09,95 \%$ CI 1.01 to $1.17, \mathrm{p}=0.04$ and $73 \%$ vs $65 \%$, RR $1.33,95 \%$ CI 1.02 to $1.26, \mathrm{p}=0.02$, respectively) (table 3). Those who collectively received all immunisations within a week of the expected date were also significantly higher in the intervention compared with the control group (57\% vs 47\%, RR 1.22, $95 \%$ CI 1.04 to 1.43; $p=0.01$ ) (table 3 ). In the post hoc subgroup analysis, there was no difference in the immunisation rates by the selected demographic variables assessed (table 4).

\section{Feasibility, acceptability of intervention and barriers to completing immunisation assessed during the mid-study survey}

At enrolment, 277 (92\%) of those in the intervention group received a confirmatory text and call. Failed delivery resulted from poor local telecommunication service. It affected either internet or mobile phone connectivity. The mid-study survey was completed by $276(92 \%)$ and $281(94 \%)$ subjects in the control and intervention groups, respectively. In the intervention group, $86 \%$ reported they received reminders at least once during the study period. Twenty-five mothers (13\%) reported receiving a partial or an unclear text reminder. Sixty-six per cent thought the text and calls were overall useful in reminding them of their child's appointment and $78 \%$ reported it reminded them all or some of the time of appointment dates.

Based on the survey data, the biggest reported barriers to completing immunisations were long wait times in 308 $(55 \%)$ and transportation cost in $187(34 \%)$. Forgetfulness $(12 ; 2.2 \%)$ was one of the least reported barriers to completing the routine immunisation series (online supplementary tables 3 and 4 ).

\section{DISCUSSION}

Paired automated calls with text immunisation reminders significantly improved the proportion of infants who completed all routine immunisations by 12 months of age and the timeliness of vaccines administered late in 
Table 1 Infant and maternal demographics by study group

\begin{tabular}{|c|c|c|c|}
\hline & Control $(n=300)$ & $\begin{array}{l}\text { Intervention } \\
(\mathrm{n}=300)\end{array}$ & Total $(\mathrm{N}=600)$ \\
\hline \multicolumn{4}{|l|}{ Infant demographics } \\
\hline Birth weight, g, mean (SD) & $3081.0(505.5)$ & $3118.7(463.1)$ & $3099.8(484.7)$ \\
\hline Gestational age, weeks, median (IQR) & $38(37-38)$ & $38(37-38)$ & $38(37-38)$ \\
\hline Married, n (\%) & $292(97)$ & 297 (99) & $589(98)$ \\
\hline \multicolumn{4}{|l|}{ Maternal education† } \\
\hline University & $163(54.3)$ & $183(61.0)$ & $346(57.7)$ \\
\hline - Secondary & $123(41.0)$ & $109(36.3)$ & $232(38.7)$ \\
\hline - Professional, top civil servant, politician or businesswoman & $116(38.7)$ & $110(37.0)$ & $226(37.9)$ \\
\hline $\begin{array}{l}\text { Middle bureaucrats, technicians, skilled artisans, well-to-do } \\
\text { trader }\end{array}$ & $70(23.3)$ & $75(25.3)$ & $145(24.3)$ \\
\hline - Unskilled worker & $60(20.0)$ & $56(18.9)$ & $116(19.4)$ \\
\hline Housewife, unemployed & $54(18.0)$ & $56(18.9)$ & $110(18.4)$ \\
\hline Christian, n (\%) & $279(93.0)$ & $280(93.3)$ & $559(93.2)$ \\
\hline \multicolumn{4}{|l|}{ No of children age $\leq 5, \mathrm{n}(\%)$} \\
\hline$>1$ & $149(50.5)$ & $164(54.8)$ & $313(52.7)$ \\
\hline$>2$ & $108(36.6)$ & $111(37.1)$ & $219(36.9)$ \\
\hline - Walk & $13(4.3)$ & $8(2.7)$ & $21(3.5)$ \\
\hline - Public transportation & $239(79.7)$ & $237(79.0)$ & $476(79.3)$ \\
\hline - Personal car & $48(16.0)$ & $55(18.3)$ & $103(17.2)$ \\
\hline $\begin{array}{l}\text { Average cost of transportation to immunisation clinic (US\$), } \\
\text { median (IQR) }\end{array}$ & $0.6(0.4-0.9)$ & $0.6(0.4-0.9)$ & $0.6(0.4-0.9)$ \\
\hline Average time to the hospital (min), median (IQR) & $20(12.5-30)$ & $20(10-25)$ & $20(10-25)$ \\
\hline \multicolumn{4}{|l|}{ Report viewing or hearing advertisements about immunisations, n (\%) } \\
\hline Most times (at least twice a week) & $152(50.8)$ & $165(55.0)$ & $317(52.9)$ \\
\hline Sometimes (at least twice a month/<twice a week) & $118(39.5)$ & $111(37.0)$ & $229(38.2)$ \\
\hline Never & $29(9.7)$ & $24(8.0)$ & $53(8.8)$ \\
\hline \multicolumn{4}{|l|}{ Mother's knowledge about and attitude towards immunisations, n (\%) } \\
\hline Agree, immunisations prevent disease (agree) & $295(98.3)$ & $296(98.7)$ & $591(98.5)$ \\
\hline Agree, immunisation prevents diseases that can lead to death & $245(81.7)$ & $247(82.3)$ & $492(82.0)$ \\
\hline Disagree, immunisations harmful to child & $292(97.3)$ & $290(97.0)$ & $582(97.2)$ \\
\hline Knew immunisations are completed within 1 year of life & $245(82.2)$ & $251(83.7)$ & $496(82.9)$ \\
\hline Able to name two diseases immunisation prevents & $170(56.7)$ & $184(61.3)$ & $354(59.0)$ \\
\hline \multicolumn{4}{|l|}{ Mother's health and health-seeking behaviours, n (\%) } \\
\hline Rates health as good & $295(98.3)$ & $290(96.7)$ & $585(97.5)$ \\
\hline
\end{tabular}


Table 1 Continued

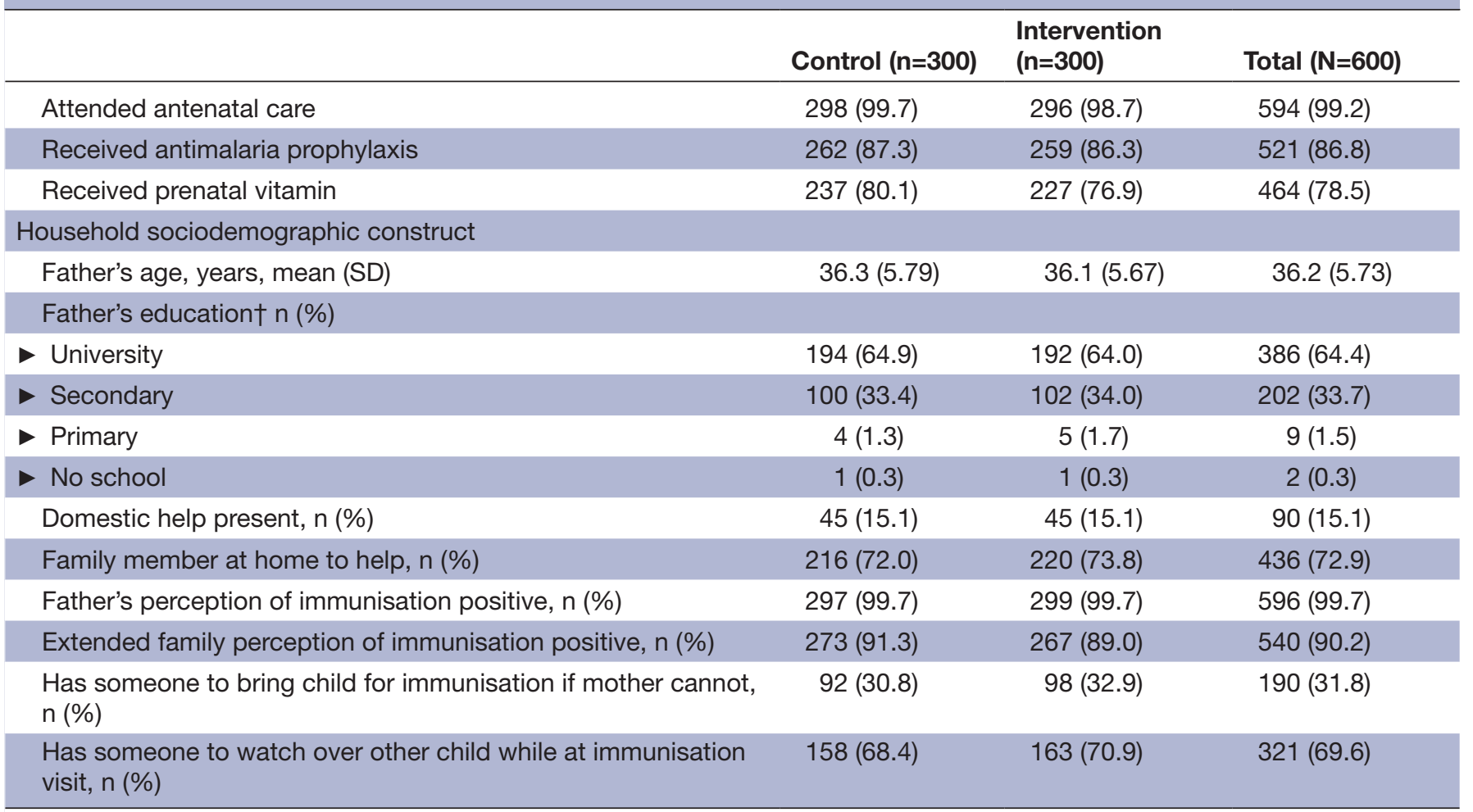

${ }^{*}$ A director or manager of a government entity is an example of a top civil servant, while a clerk, typist or cashier is an example of a middle bureaucrat.

†Educational level indicates having some or completed the level of education.

the immunisation schedule. For the primary outcome, however, Penta-3 completion rates were higher than anticipated in both groups and not significantly different between groups.

For the Penta-3 completion, our findings differ from trials where text messages reminders alone were used in urban SSA settings, ${ }^{7-9}$ but are similar to a trial in rural Kenya. ${ }^{10}$ This may indicate that low literacy may not account for the lack of effectiveness of text reminders. An inappropriate study population may also explain this difference. Although we did not assess literacy level directly, $58 \%$ and $39 \%$ of our study participants had at least some university or secondary education, respectively.

Lack of 'awareness' ranks highest among barriers to immunisation completion reported by the Nigerian National Immunization Survey. ${ }^{5}$ The relatively high awareness among the standard of care group in our study may reflect above-average site-specific counselling. Cost of transportation, distance to and overall 'conduciveness' at the clinics were the most frequent barriers to immunisation in our study population. These barriers have also been reported in trials conducted in Kenya and Nigeria. ${ }^{822}$ Furthermore, they have attracted research interest from organisations like the Bill and Melinda Gates foundation in recent years. ${ }^{23}$

Findings from a study in rural Nigeria where realtime phone call immunisation reminders significantly improved immunisation completion premised our intent to use automated calls. ${ }^{14}$ The Nigeria study found a $41 \%$ increase in immunisation completion. ${ }^{14}$ Our intervention also resulted in improvements in immunisation completion at 12 months, with a smaller effect. Methodological

\begin{tabular}{llllll}
\hline Table 2 & Effect of Intervention on primary and secondary outcomes by study group* & & \\
\hline Immunisation & Control $(\mathbf{n}=\mathbf{3 0 0})$ & Intervention $(\mathbf{n}=\mathbf{3 0 0})$ & $\mathbf{R R}(\mathbf{9 5 \%} \mathbf{C l})$ & RD (95\% Cl) & P value \\
\hline Penta-1† $\mathrm{n}(\%)$ & $289(97)$ & $285(95)$ & $0.98(0.95$ to 1.02$)$ & $-0.02(-0.05$ to 0.015$)$ & 0.31 \\
Penta-2† $\mathrm{n}(\%)$ & $278(93)$ & $276(92)$ & $0.99(0.95$ to 1.04$)$ & $-0.01(-0.04$ to 0.05$)$ & 0.76 \\
\hline Penta-3† $\mathrm{n}(\%)$ & $244(81)$ & $257(86)$ & $1.05(0.98$ to 1.13$)$ & $0.04(-0.02$ to 0.10$)$ & 0.15 \\
\hline Measles $\mathrm{n}(\%)$ & $196(66)$ & $220(74)$ & $1.12(1.01$ to 1.25$)$ & $0.08(0.01$ to 0.15$)$ & 0.03 \\
\hline
\end{tabular}

*Modified intention-to-treat analysis; includes all subjects regardless of where immunisation was received or how it was audited. †Assessed at 18 weeks.

$\ddagger$ Assessed at 12 months.

$\mathrm{RD}$, risk difference; $\mathrm{RR}$, risk ratio. 
Table 3 Timely receipt of immunisation -individually and collectively by study group ${ }^{\star} \dagger$

\begin{tabular}{lllll}
\hline Immunisation $\mathbf{n}(\%)$ & Control $(\mathbf{n = 3 0 0 )}$ & Intervention $(\mathbf{n = 3 0 0 )}$ & RR (95\% Cl) & P value \\
\hline Penta-1 at 6 weeks & $250(83)$ & $257(86)$ & $1.03(0.96$ to 1.10$)$ & 0.43 \\
Penta-2 at 10 weeks & $252(84)$ & $256(85)$ & $1.02(0.95$ to 1.09$)$ & 0.65 \\
Penta-3 at 14 weeks & $233(78)$ & $253(84)$ & $1.09(1.01$ to 1.17$)$ & 0.04 \\
Measles at 9 months & $194(65)$ & $220(73)$ & $1.13(1.02$ to 1.26$)$ & 0.02 \\
All immunisations & $140(47)$ & $171(57)$ & $1.22(1.04$ to 1.43) & 0.01 \\
\hline
\end{tabular}

*Modified intention-to-treat analysis. Includes all subjects regardless of where immunisation was received or how it was audited.

†Timeliness defined as receiving vaccinations within 1 week of their due date.

$R R$, risk ratio.

differences may explain effect size differences. While our study sent out two reminder calls 2 days and the day before the immunisation due date, the prior published study used two real-time reminder phone calls plus four recalls if immunisations were missed as the primary intervention. ${ }^{14}$ Furthermore, and likely of importance, is the absence of the human element in automated calls. The ability to empathise, counsel and reassure mothers during a real-time phone call is lost with automation.
However, with current resource and organisational limitations, real-time phone calls plus recalls is time and human resource dependent and likely to result in a high implementation cost. Taking it to scale and sustenance will also be a challenge. Since most studies where text was used yielded between $60 \%$ and $80 \%$ completion rates, automated text and/or calls could serve as a first-pass reminder to be followed by real-time phone calls when immunisations are delayed. This may prove to be a more

Table 4 Subgroup analysis of completing immunisation by 12 months of age by study group

\begin{tabular}{|c|c|c|c|c|}
\hline Characteristic & Control $(n=300)(\%)$ & $\begin{array}{l}\text { Intervention }(\mathrm{n}=300) \\
(\%)\end{array}$ & $\begin{array}{l}\text { Stratum-specific RR } \\
(95 \% \mathrm{Cl})\end{array}$ & $P$ value \\
\hline $\begin{array}{l}\text { Sex } \\
\text { Male } \\
\text { Female }\end{array}$ & $\begin{array}{l}112 / 163(69) \\
86 / 137(63)\end{array}$ & $\begin{array}{l}116 / 148(78) \\
105 / 152(69)\end{array}$ & $\begin{array}{l}1.14(1.00 \text { to } 1.30) \\
1.10(0.93 \text { to } 1.30)\end{array}$ & 0.74 \\
\hline $\begin{array}{l}\text { Maternal education } \\
\text { - Completed university } \\
\text { Did not complete university }\end{array}$ & $\begin{array}{l}124 / 163(76) \\
74 / 137(54)\end{array}$ & $\begin{array}{l}144 / 183(79) \\
77 / 117(66)\end{array}$ & $\begin{array}{l}1.22(1.00 \text { to } 1.49) \\
1.03(0.92 \text { to } 1.16)\end{array}$ & 0.17 \\
\hline $\begin{array}{l}\text { Mothers age (years) } \\
>>35 \\
\leq 35\end{array}$ & $\begin{array}{l}28 / 40(70) \\
169 / 259(65)\end{array}$ & $\begin{array}{l}22 / 30(73) \\
199 / 270(74)\end{array}$ & $\begin{array}{l}1.05(0.78 \text { to } 1.41) \\
1.13(1.01 \text { to } 1.27)\end{array}$ & 0.21 \\
\hline $\begin{array}{l}\text { No of children under age } 5 \\
->1 \\
>\leq 1\end{array}$ & $\begin{array}{l}94 / 146(64) \\
101 / 149(68 \%)\end{array}$ & $\begin{array}{l}104 / 135(77) \\
117 / 164(71 \%)\end{array}$ & $\begin{array}{l}1.20(1.03 \text { to } 1.39) \\
1.05(0.91 \text { to } 1.22)\end{array}$ & 0.23 \\
\hline $\begin{array}{l}\text { Mother owns a car } \\
\text { No } \\
\text { Yes }\end{array}$ & $\begin{array}{l}180 / 275(65) \\
18 / 25(72)\end{array}$ & $\begin{array}{l}192 / 263(73) \\
29 / 37(78)\end{array}$ & $\begin{array}{l}1.12(1.00 \text { to } 1.25) \\
1.09(0.81 \text { to } 4.47)\end{array}$ & 0.88 \\
\hline $\begin{array}{l}\text { Mother's mode of transportation } \\
\text { - Public transportation or walk } \\
\text { - Personal car }\end{array}$ & $\begin{array}{l}171 / 252(68) \\
27 / 48(56)\end{array}$ & $\begin{array}{l}183 / 245(75) \\
38 / 55(69)\end{array}$ & $\begin{array}{l}1.10(0.98 \text { to } 1.23) \\
1.23(0.91 \text { to } 1.67)\end{array}$ & 0.51 \\
\hline $\begin{array}{l}\text { Able to name two diseases } \\
\text { immunisation prevents } \\
\text { No } \\
\text { Yes }\end{array}$ & $\begin{array}{l}64 / 92(70) \\
134 / 208(640\end{array}$ & $\begin{array}{l}57 / 82(70) \\
164 / 218(75)\end{array}$ & $\begin{array}{l}1.00(0.98 \text { to } 1.23) \\
1.23(0.91 \text { to } 1.67)\end{array}$ & 0.51 \\
\hline $\begin{array}{l}\text { Someone to bring child for } \\
\text { immunisation if mother cannot } \\
\text { No } \\
\text { Yes }\end{array}$ & $\begin{array}{l}145 / 207(70 \%) \\
53 / 92(58 \%)\end{array}$ & $\begin{array}{l}151 / 200(76 \%) \\
70 / 98(71 \%)\end{array}$ & $\begin{array}{l}1.08(0.96 \text { to } 1.21) \\
1.24(1.00 \text { to } 1.15)\end{array}$ & 0.27 \\
\hline $\begin{array}{l}\text { Study site location } \\
\text { Ondo Town } \\
\text { Akure }\end{array}$ & $\begin{array}{l}59 / 125(47 \%) \\
139 / 175(79 \%)\end{array}$ & $\begin{array}{l}73 / 125(58 \%) \\
148 / 175(85 \%)\end{array}$ & $\begin{array}{l}1.24(0.98 \text { to } 1.57) \\
1.06(0.96 \text { to } 1.18)\end{array}$ & 0.25 \\
\hline
\end{tabular}

Data are $\mathrm{n} / \mathrm{N}(\%)$ and $\mathrm{RR}(95 \% \mathrm{Cl})$. P values obtained from an interaction term between intervention.

$\mathrm{RR}$, risk ratio. 
cost-effective strategy. Digital immunisation registries can potentially improve the efficiency of this process by simplifying identification of defaulters.

In our study, we demonstrated modest feasibility instituting a customised automated text and call immunisation reminder system. However, poor phone and internet connectivity resulted in $8 \%$ delivery failure at enrolment. Furthermore, only $86 \%$ reported receiving at least one set of reminders during the study period. Phones that were switched off or had poor reception when messages were sent were the most common reported reasons for not receiving phone reminders. This is a limitation in web-based text and call systems which lack the ability to queue messages until the switched off phone is turned back on or a phone in poor reception areas moves to an area with better reception. In addition, voicemail is uncommon in these settings. We speculate that a system that uses the available local telecommunication network, which is usually more redundant, and has the capacity to provide more accurate log data, may yield better results.

Our study had some limitations. A 2015 institutional audit and state reported Penta-3 completion rates informed our sample size estimation. This likely underestimated the true institutional completion for those committed to receiving their immunisations at only $\mathrm{MCH}$ clinic. Furthermore, we introduced selection bias by recruiting only parents who planned for their child to receive immunisations only at $\mathrm{MCH}$. Ondo state government during the study period provided free maternal care. Women in lower socioeconomic classes may have been those who lived further away and only used MCH for its free services. However, this was necessary to allow us to ascertain our intended primary outcome (immunisations confirmed by clinic records). The inclusion of women who own phones limits the generalisation of our results. Another limitation was the workers' strike. Unfortunately, this reflects a common reality in SSA and a need to strengthen the healthcare system. We were, however, able to retain $92 \%$ of the enrolled population for the primary outcome. Due to a clerical error, the number of subjects who refused or were ineligible for the study at MCH-Ondo could not be determined (figure). Another limitation is our inability to access reminder messaging $\log$ from our web-based platform. This is a limitation in most similar trials from SSA with only one trial reporting $\log$ data. ${ }^{10}$ Finally, although we intended to overcome low literacy levels by adding voice call reminders, $97 \%$ of study participants had at least secondary education.

The strengths of our study include a randomisation strategy that resulted in balanced demographic characteristics between study groups. We also used a more practical approach to study participant's message receipt as a proxy to assess feasibility.

\section{CONCLUSIONS}

In a semi-rural region in Nigeria, the implementation of an automated text and call immunisation system was modestly feasible. The phone reminders significantly improved immunisation completion and timeliness. This study adds to the growing evidence of the effectiveness of phone reminders in SSA. Factors such as transportation cost, distance to and overall 'immunisation clinic conduciveness' were the most frequently reported barriers to immunisation. Combining reminder systems with innovative solutions capable of improving access to and efficiency of immunisation clinics are likely to improve immunisation uptake and completion in Nigeria and other SSA countries.

\section{Author affiliations}

${ }^{1}$ Neonatal-Perinatal Medicine, Department of Pediatrics, Indiana University, Indianapolis, Indiana, United States

${ }^{2}$ Department of Pediatrics, Mother and Child Hospital, Akure, Ondo State, Nigeria ${ }^{3}$ Department of Pediatrics, Mother and Child Hospital, Ondo Town, Ondo State, Nigeria

${ }^{4}$ Department of Obstetrics and Gynecology, Mother and Child Hospital, Ondo Town, Ondo State, Nigeria

${ }^{5}$ Department of Oral Pathology, Radiology and Medicine, College of Dentistry, University of lowa, lowa City, lowa, USA

${ }^{6}$ Pediatrics and Epidemiology, Perelman School of Medicine, Philadelphia,

Pennsylvania, USA

${ }^{7}$ Division of Infectious Diseases, The Children's Hospital of Philadelphia, Philadelphia, PA, USA

Acknowledgements We thank the mothers and infants who participated in this study. The authors wish to thank the Ondo State Ministry of Health and The Mother and Child Hospitals. The authors also thank the research assistants on this project, Busola Akingboye and Tosin Akinrinola. We thank Okan Elci for performing the statistics and Akinwande Benka-Coker for additional statistical support. We would also like to thank Priscilla Dikko for her administrative duties and Abdulwahab Suleiman for developing and refining the customised software used for this study. We wish to thank the Christiansen Professorship for Global Oral Health at the University of lowa for the support provided to conduct the study to develop the text and voice message content. Special thanks to all facilitators at the nominal group technique meeting. We appreciate Sara Demuaro for randomisation sequence generation and Eric Jensen and Heather French for the technical advice they provided.

Contributors $\mathrm{OAE}$ and $\mathrm{AB}$ conceptualised the study. OAE developed the study design, study protocol, performed research assistant training, implemented the project and wrote the first draft. AS and EL provided critical technical assistance in study design, protocol development, project implementation and manuscript review. AS and EL contributed equally as senior authors. ROO and BB were local site PIs. They coordinated all aspects of the study; they provided oversight and supervision of the research assistants. ROO, AB and LO provided administrative assistance for the study and provided critical review of the manuscript.

Funding The study was funded by the Thrasher Research Fund (award no. 13813). OAE was awarded a Thrasher Early Career Development Grant as a neonatal-perinatal medicine fellow at the Children's Hospital of Philadelphia. The corresponding author had full access to all the data in the study and had final responsibility for the decision to submit for publication.

Disclaimer The funder of the study had no role in study design, data collection, data analysis, data interpretation or writing of the report.

\section{Competing interests None declared.}

Patient consent for publication Obtained.

Ethics approval Obtained from the Health Research Ethics Board of the Mother and Child Hospitals, the Ondo State Ministry of Health and the Children's Hospital of Philadelphia Institutional Review Board.

Provenance and peer review Not commissioned; externally peer reviewed.

Data sharing statement All available data can be obtained by contacting the corresponding author. All data requests should be submitted to the corresponding author for consideration. Access to anonymised data may be granted following review. 
Open access This is an open access article distributed in accordance with the Creative Commons Attribution Non Commercial (CC BY-NC 4.0) license, which permits others to distribute, remix, adapt, build upon this work non-commercially, and license their derivative works on different terms, provided the original work is properly cited, appropriate credit is given, any changes made indicated, and the use is non-commercial. See: http://creativecommons.org/licenses/by-nc/4.0/.

\section{REFERENCES}

1. Liu L, Oza S, Hogan D, et al. Global, regional, and national causes of under-5 mortality in 2000-15: an updated systematic analysis with implications for the sustainable development goals. Lancet 2016;388:3027-35.

2. Hug L, Sharrow D, You D. Estimates developed by the UN Interagency group for child mortality estimation, 2017.

3. WHO, 2018. Data, statistics and graphics. Available: http://www. who.int/immunization/monitoring_surveillance/data/en/ [Accessed 7 Jun 2018].

4. WHO, 2018. Immunization coverage. Available: http://www.who.int/ en/news-room/fact-sheets/detail/immunization-coverage [Accessed 24 Apr 2018].

5. IVAC, 2018. Nigeria national immunization coverage survey 2016-2017. Available: https://www.jhsph.edu/research/centers-andinstitutes/ivac/resources/Nigeria-National-Immunization-CoverageSurvey [Accessed 22 Apr 2018].

6. Jacobson Vann JC, Jacobson RM, Coyne-Beasley T, et al. Patient reminder and recall interventions to improve immunization rates. Cochrane Database Syst Rev 2018;1.

7. Bangure D, Chirundu D, Gombe N, et al. Effectiveness of short message services reminder on childhood immunization programme in Kadoma, Zimbabwe-a randomized controlled trial, 2013. BMC Public Health 2015;15:137-015.

8. Eze GU, Adeleye OO. Enhancing routine immunization performance using innovative technology in an urban area of Nigeria. West Afr $J$ Med 2015;34:3-10.

9. Schlumberger M, Bamoko A, Yaméogo TM, et al. Impact positif sur le Programme élargi de vaccinations de l'envoi de SMS de rappel à partir d'un registre informatisé, Bobo-Dioulasso (Burkina Faso). Bulletin de la Société de pathologie exotique 2015;108:349-54.

10. Gibson DG, Ochieng B, Kagucia EW, et al. Mobile phone-delivered reminders and incentives to improve childhood immunisation coverage and timeliness in Kenya (M-SIMU): a cluster randomised controlled trial. The Lancet Glob Health 2017;5:e428-38.

11. Literacy United Nations Educational. Scientific and cultural organization. Available: //www.unesco.org/new/en/dakar/education/ literacy [Accessed 27 Jul 2018].

12. Coulombe H, Lanjouw P. Poverty, access to services and city size in a selection of African countries. Washington, DC: World Bank, 2013mimeo..

13. Zhang Y. Urban-rural literacy gaps in sub-Saharan Africa: the roles of socioeconomic status and school quality. Comp Educ Rev 2006;50:581-602.

14. Brown VB, Oluwatosin OA, Akinyemi JO, et al. Effects of community health nurse-led intervention on childhood routine immunization completion in primary health care centers in Ibadan, Nigeria. $J$ Community Health 2016;41:265-73.

15. Moher D, Hopewell S, Schulz KF, et al. Consort 2010 explanation and elaboration: updated guidelines for reporting parallel group randomised trials. Int J Surg 2012;10:28-55.

16. Ophori EA, Tula MY, Azih AV, et al. Current trends of immunization in Nigeria: prospect and challenges. Trop Med Health 2014;42:67-75.

17. Ondo Statestate Bureau of Statistics, 2018. Home page. Available: http://www.ondostatistics.org/ondo_profile.php [Accessed 11 Jul 2018].

18. WHO, 2018. Vaccine position papers. Available: http://www.who. int/immunization/documents/positionpapers/en/ [Accessed 10 Oct 2018].

19. Matts JP, Lachin JM. Properties of permuted-block randomization in clinical trials. Control Clin Trials 1988;9:327-44.

20. Adedokun ST, Uthman OA, Adekanmbi VT, et al. Incomplete childhood immunization in Nigeria: a multilevel analysis of individual and contextual factors. BMC Public Health 2017;17.

21. Antai D. Inequitable childhood immunization uptake in Nigeria: a multilevel analysis of individual and contextual determinants. BMC Infect Dis 2009;9.

22. Haji A, Lowther S, Ngan'ga Z, et al. Reducing routine vaccination dropout rates: evaluating two interventions in three Kenyan districts, 2014. BMC Public Health 2016;16:152-016.

23. Global Grand Challenges. Innovations in immunization data management, use, and improved process efficiency (round 21) grand challenges. Available: gcgh.grandchallenges.org/challenge/ innovations-immunization-data-management-use-and-improvedprocess-efficiency-round-21 [Accessed 9 Sep 2018] 